\title{
Modeling, Analysis, and Optimal Design of the Automotive Transmission Ball Capsule System
}

\author{
Xingyong Song, Mohd Azrin Mohd Zulkefli, Zongxuan Sun, and Hsu-Chiang Miao
}

\begin{abstract}
Clutch shift control is critical for the performance and fuel economy of automotive transmission, including both automatic and hybrid transmissions. Among all the factors that influence clutch shift control, clutch fill and clutch engagement are crucial to realize a fast and smooth clutch shift. When the clutch is not engaged, the fluid held by the centrifugal force inside of the clutch chamber, which introduces additional pressure that will affect the subsequent clutch fill and engagement processes, should be released. To realize this function, a ball capsule system is introduced and mounted on the clutch chamber. When the clutch is ready to be filled for engagement, the ball capsule needs to close quickly and remain closed until the clutch is disengaged. It's also desirable to have appropriate closing velocity for the ball capsule to minimize noise and wear. In this paper, we will model the ball capsule dynamics and reveal its intrinsic positive feedback structure, which is considered to be the key to realize a fast response, and design the optimal shape of the capsule to achieve the desired performance.
\end{abstract}

\section{INTRODUCTION}

$\mathrm{T}_{\mathrm{e}}^{\mathrm{o}}$ o reduce vehicle fuel consumption and tailpipe emissions, automotive manufacturers have been developing new technologies for powertrain systems. In the transmission area, emerging technologies $[1,2]$ such as the six or more speeds automatic transmission (AT), the continuously variable transmission (CVT), the dual clutch transmission (DCT), the automated manual transmission (AMT), and the electrically variable transmission (EVT) have appeared in the market.

Recently with the introduction of the six or more speeds automatic transmissions, the clutch to clutch shift control technology [3-6] has once again attracted a lot of researches and development efforts. This clutch shift control technology is the key enabler for a compact, light, and low cost automatic transmission design. This technology uses pressure control valves to control the clutch engagement and disengagement processes of the oncoming and the off-going clutches. A critical challenge for clutch shift control is the synchronization of the oncoming and off-going clutches, which is highly dependent on the oncoming clutch fill time [13].

This work was supported in part by General Motors Research and Development Center.

Xingyong Song, Mohd Azrin Mohd Zulkefli, and Zongxuan Sun are with the Mechanical Engineering Department, University of Minnesota, Twin Cities, Minneapolis, MN 55455. (email: zsun@umn.edu).

Hsu-Chiang Miao is with Research and Development Center, General Motors Corporation, Warren, MI 48090.
A schematic diagram of the transmission clutch system is shown in Figure 1. To control the clutch fill precisely, it is desirable that the initial condition of the clutch fill process does not change from cycle to cycle. However, since the whole clutch system rotates around the shaft (Figure 1), the centrifugal force will keep certain amount of fluid at the ceiling of the clutch chamber. The fluid pressure induced by the rotation of the leftover fluid will push the piston and subsequently affect the initial conditions of the clutch fill process in the following cycle. To dissipate the leftover fluid and subsequently release the centrifugal force induced pressure, a ball capsule system is introduced and mounted on the clutch chamber as shown in Figure 1.

The ball capsule system consists of a ball and a capsule. Together with the whole clutch system, the capsule rotates around the central shaft (Figure 1). The centrifugal force of the ball keeps it in contact with the capsule ceiling, so the ball can rotate along the capsule inner wall between the open position and the closed position. The opening and closing of the ball capsule system is controlled by the fluid pressure inside the clutch chamber and the ball's centrifugal force. When the fluid pressure inside the chamber is below a specific level, the sum of moments acting on the ball around the contact point will make the ball rotate along the capsule inner wall from the closed position to the open position. The fluid inside the clutch chamber then can flow out to the exhaust through the opening area between the ball and the inner wall of the capsule. On the other hand, when the fluid pressure inside the chamber is high enough, the ball will rotate to the closed position and seal off the exhaust port.

The performance of the ball capsule system is crucial for clutch engagement and disengagement processes. As shown in Figure 1, when clutch disengages, the piston is pushed to the left by the spring force, and the fluid inside the clutch chamber is dissipated through the inlet orifice. At this time, the pressure inside the clutch chamber drops, and the ball capsule needs to open to allow the fluid held by the centrifugal force at the ceiling of the chamber to flow out to the exhaust. When the clutch fill process starts, the pressurized fluid enters the clutch chamber through the inlet orifice. When this happens, the ball needs to close quickly in order to build up the pressure inside of the chamber. In addition to these requirements, the system also needs to be robust and able to avoid undesirable ball chattering between the open and closed positions. In this paper, we will model the dynamics of the ball capsule system, analyze the stability 
of the system, and provide the optimal capsule design to achieve the desired performance.

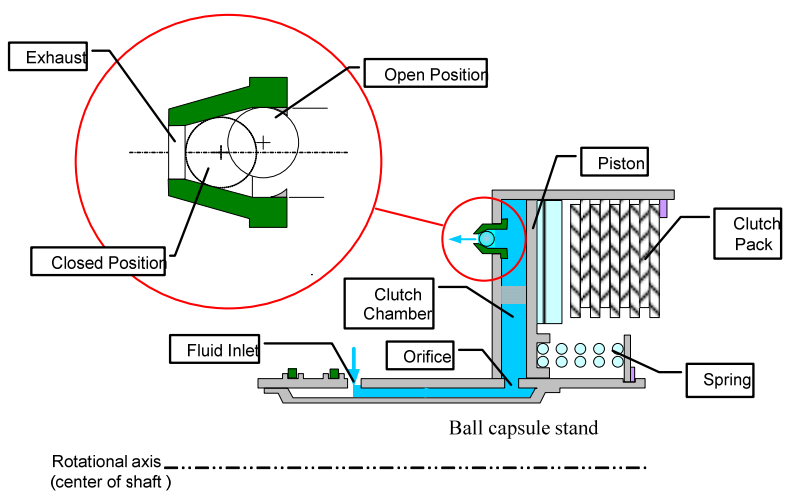

Fig 1. Clutch and ball capsule system

The rest of the paper is organized as follows. Section 2 presents the system dynamic model. Section 3 analyzes the intrinsic positive feedback structure of the ball capsule system. Section 4 formulates the ball capsule design problem as an optimization problem. To this end, the Dynamic Programming method is applied to redesign the shape of the ball capsule inner wall. Section 5 presents case studies and simulation results.

\section{SYSTEM MODELING}

Figure 2 shows the schematic diagram of the ball capsule system.

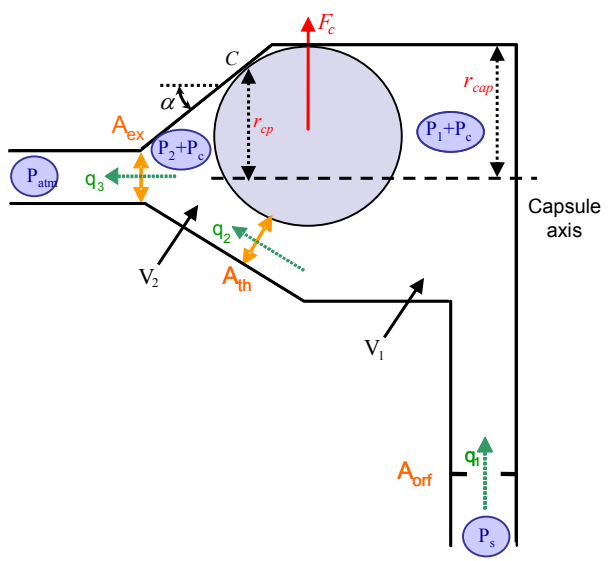

Fig 2. Ball Capsule System Model

The ball capsule system model can be expressed as:

$\dot{\theta}=v$

$J \dot{v}=\left(P_{1}+P_{c}\right) A_{e f f} r_{b} \cos (\alpha)-\left(P_{2}+P_{c}\right) A_{e f f} r_{b} \cos (\alpha)$

$-F_{c} r_{b} \sin (\alpha)$

$\dot{P}_{1}=\frac{\beta}{V_{1}}\left(q_{1}-q_{2}\right)$

$\dot{P}_{2}=\frac{\beta}{V_{2}}\left(q_{2}-q_{3}\right)$

where

$$
\begin{gathered}
q_{1}=C_{d} A_{\text {orf }} \sqrt{\frac{2}{\rho_{\text {oil }}}\left(P_{S}-P_{1}\right)} \\
q_{2}=C_{d} A_{t h} \sqrt{\frac{2}{\rho_{\text {oil }}}\left(P_{1}-P_{2}\right)} \\
q_{3}=C_{d} A_{\text {ex }} \sqrt{\frac{2}{\rho_{\text {oil }}}\left(P_{2}+P_{c}-P_{\text {atm }}\right)} \\
A_{\text {eff }}=\pi\left(r_{b} \cos (\alpha)\right)^{2} \\
F_{c}=\frac{4}{3} \pi r_{b}^{3}\left(\rho_{\text {steel }}-\rho_{\text {oil }}\right) r_{c} \omega^{2} \\
r_{c p}=r_{\text {cap }}-r_{b}+r_{b} \cos (\alpha)-r_{b} \theta \sin (\alpha)
\end{gathered}
$$

$$
\begin{aligned}
A_{t h}= & \pi \cdot\left(r_{b}+\frac{x_{l i f t}}{2} \sin \alpha\right) \sin (2 \alpha) \cdot x_{l i j t} \\
& +\pi \cdot\left(1-\frac{\sin ^{2} \alpha}{2}\right) \sin \alpha \tan \alpha \cdot x_{l i f t}^{2} \\
& -\frac{\pi\left(x_{l i f t} \tan \alpha\right)^{2}}{2 r_{b} \cos \alpha}\left(x_{l i f t}+\frac{r_{b}}{\sin \alpha}\right) \sin (2 \alpha) \cos (\alpha)
\end{aligned}
$$

$$
\begin{aligned}
x_{l i f t} & =\frac{\left[r_{c p}-r_{b} \times \cos (\alpha)\right]}{\tan (\alpha)} \\
& =\frac{\left[r_{c a p}-r_{b}+r_{b} \cos (\alpha)-r_{b} \theta \sin (\alpha)-r_{b} \times \cos (\alpha)\right]}{\tan (\alpha)}
\end{aligned}
$$

where $\theta$ is the rotational angle of the ball, $v$ is the ball angular velocity, $r_{b}$ is the radius of the ball, and $\alpha$ is the angle of the capsule. $P_{1}$ and $P_{2}$ are the chamber pressure at opposite sides of the ball, and $P_{s}$ is the input pressure. $A_{\text {eff }}$ is the effective area, which is defined as the net area used to calculate the forces acting on the ball due to $P_{1}$ and $P_{2} P_{c}$ is the centrifugal fluid pressure due to capsule rotation and $F_{c}$ is the centrifugal force of the ball. $\omega$ is the clutch system rotational speed, $r_{c}$ is the distance from the center of the ball to the axis of the transmission, $r_{c p}$ is the distance between the contact point $C$ and the capsule axis, and $r_{c a p}$ is the capsule radius shown in Figure 2. The throttling area $A_{t h}$ is the smallest opening area between the ball and the capsule inner wall, which changes with the motion of the ball. Most of the throttling areas in fluid system modeling are symmetric [7]. However, in the ball capsule system, the derivation of the throttling area $A_{t h}$ between the ball and the capsule inner surface is not straightforward because the ball is not concentric with the conical capsule. The derivation of $A_{t h}$ will not be shown in this paper due to space limitation.

By now, we have completed the dynamic modeling for the ball capsule system. However, the Dynamic Programming method, which will be used for the capsule inner wall profile design later would suffer from heavy computational burden if the order of the system is high. Thus, it is desirable to reduce 
the order of the model while still capturing the main dynamics of the system. Since the bulk modulus of the fluid is significantly larger compared to the fluid volume, it is reasonable to assume that the volumetric flow rates are the same across the whole capsule from the orifice to the exhaust $\left(q_{1}=q_{2}=q_{3}\right)$, resulting in a second order model which can be expressed as:

$$
\begin{aligned}
& \dot{\theta}=v \\
& J \dot{v}=\left(P_{1}-P_{2}\right) A_{e f f} r_{b} \cos (\alpha)-F_{c} r_{b} \sin (\alpha)
\end{aligned}
$$

where

$$
\begin{gathered}
P_{1}=P_{s}-\frac{1}{\left[1+\left(\frac{A_{o r f}}{A_{e x}}\right)^{2}\left(1+\frac{A_{e x}^{2}}{A_{t h}^{2}}\right)\right.}\left[\left(P_{s}-P_{a t m}+P_{c}\right)\right] \\
P_{2}=\left(\frac{A_{o r f}}{A_{e x}}\right)^{2}\left(P_{s}-P_{1}\right)+P_{a t m}-P_{c}
\end{gathered}
$$

As validated by a series of simulation results (see Figure 3 ), the second order ball capsule model has been shown to be a good approximation of the fourth order ball capsule model, and therefore will be used in the following analysis and design sections.
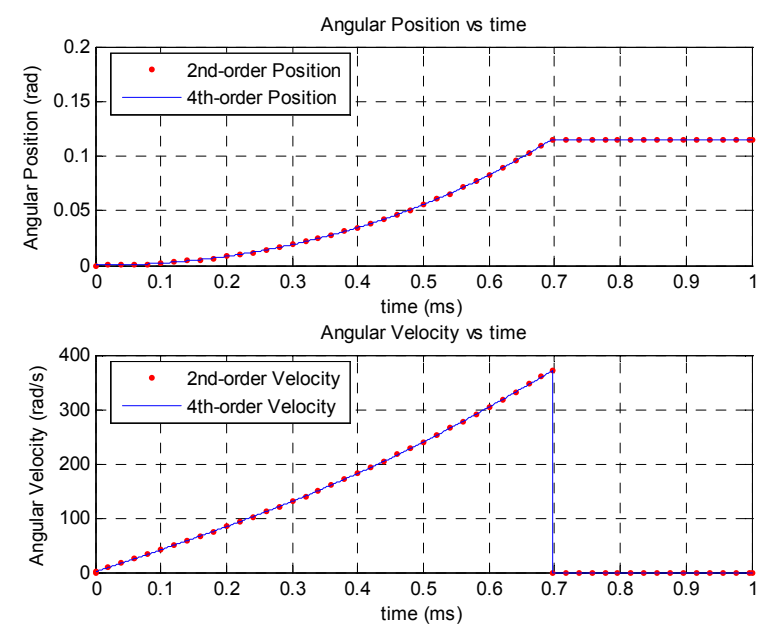

Fig 3. Simulation result comparison between 2 nd and 4 th order model

\section{SYSTEM DYNAMICS ANALYSIS}

In this section the stability of the ball capsule system is analyzed as it will provide us insight on the design of the system. The quick response of the ball capsule system is an important characteristic of the ball capsule system, which is due to an intrinsic positive feedback structure of the system. The unstable characteristic is the key to realize the quick response of the ball capsule system.

In this section we first consider the capsule with only a single slope angle, which is the simplest way to design the capsule system. Since $P_{I}$ and $P_{2}$ both depend on the throttling area $A_{t h}$, which in turn is determined by $\theta$, the ball capsule system can be represented as a closed loop feedback system shown in Figure 4.

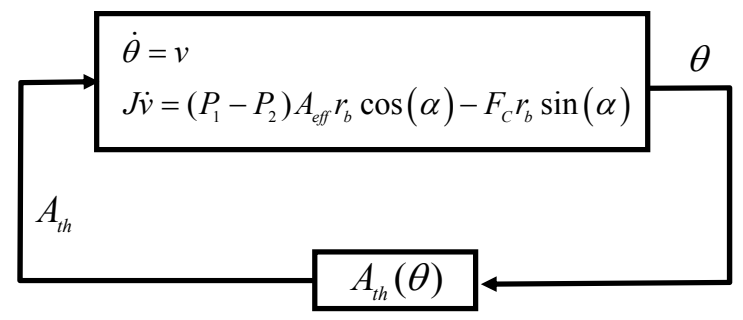

Fig. 4 System dynamics of ball capsule system

Suppose the rotational angle $\theta$ increases when the ball rotates clockwise to the exhaust, and decreases when rotating counterclockwise (see Figure 2). As $\theta$ increases, $A_{t h}$ decreases (Eq. 8 and Eq. 9), and consequently $P_{l}$ goes up (Eq. 11), while $P_{2}$ drops (Eq. 12), resulting in the rise of $\left(P_{1}-P_{2}\right)$. Furthermore, the centrifugal force $F_{c}$ will drop due to the decrease of $r_{c}$. $\quad P_{c}$ is assumed to be constant during this process. Based on the above analysis, we can see that the angular acceleration of the ball will increase (Eq. 10), which further propels the increase of $v$ and $\theta$. Therefore $v$ and $\theta$ will keep increasing until the ball reaches the exhaust port. This reveals the physical mechanism of the unstable dynamics of the ball capsule system, and a proof based on Chetaev's theorem [8] is given as follows.

Given a fixed input pressure $P_{s}$ and a fixed $\alpha$ value, the equilibrium point is supposed to be:

$$
x_{e q}=\left[\begin{array}{l}
\theta_{0} \\
0
\end{array}\right]
$$

We can assume $\theta_{0}>0$.

For the ball capsule system described by (10), a Lyapunov function is proposed

$$
V(x)=\left(\theta-\theta_{0}\right) v
$$

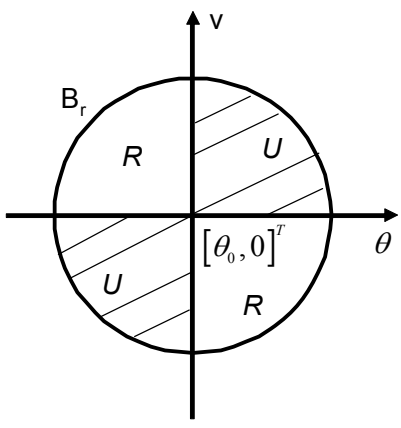

Figure 5. Region for $\mathrm{U}$

As shown in Figure 5, the circle $B_{r}$ is a region on the state space around the equilibrium point $\left[\theta_{0}, O\right]^{T}$ with a radius of $r$. Let the set $U=\left\{x \in B_{r} \mid V(x)>0\right.$ except at $\left.\left[\theta_{0}, 0\right]^{T}\right\}$, containing the first and third quadrants on the coordinate axes.

Theorem 1: For every point $x$ inside the state space except the origin $\left[\theta_{0}, 0\right]^{T}$,

$$
\dot{V}(x)=\dot{\theta} v+\dot{v}\left(\theta-\theta_{0}\right)=v^{2}+\dot{v}\left(\theta-\theta_{0}\right)>0
$$


Proof: if $\theta>\theta_{0}$, based on Equations (8) and (9), $A_{\mathrm{th}}(\theta)<$ $A_{\text {th }}\left(\theta_{0}\right)$. From Equation (11), $P_{1}(\theta)>P_{1}\left(\theta_{0}\right)$, and from Equation (12) $P_{2}(\theta)<P_{2}\left(\theta_{0}\right)$. Therefore,

$$
\left[P_{1}(\theta)-P_{2}(\theta)\right]>\left[P_{1}\left(\theta_{0}\right)-P_{2}\left(\theta_{0}\right)\right]
$$

In addition, from Equation $(10), r_{\mathrm{c}}(\theta)<r_{\mathrm{c}}\left(\theta_{0}\right)$. Then based on Equation (6),

$$
F_{c}(\theta)<F_{c}\left(\theta_{0}\right)
$$

From Equations (10), (15) and (16), we can conclude

$$
J \dot{v}(\theta)>J \dot{v}\left(\theta_{0}\right)=0
$$

Therefore

$$
\dot{v}(\theta)>0, \quad \text { and } \dot{V}(x)=\dot{\theta} v+\dot{v}\left(\theta-\theta_{0}\right)=v^{2}+\dot{v}\left(\theta-\theta_{0}\right)>0
$$

If $\theta<\theta_{0}$, using the same approach, we can get

$$
\begin{aligned}
& \dot{v}(\theta)<0, \text { and }\left(\theta-\theta_{0}\right)<0 \\
& \text { so } \dot{V}(x)=\dot{\theta} v+\dot{v}\left(\theta-\theta_{0}\right)=v^{2}+\dot{v}\left(\theta-\theta_{0}\right)>0
\end{aligned}
$$

If $\theta=\theta_{0}, \quad \dot{V}(x)=\dot{\theta} v+\dot{v}\left(\theta_{0}-\theta_{0}\right)=v^{2}>0$. Therefore, $\dot{V}(x)>0$ for every $x$ in the state space except the origin $\left[\theta_{0}\right.$, $0]^{T}$.

Therefore, in region $U$, the Lyapunov function $\mathrm{V}(\mathrm{x})>0$ and its derivative is also larger than zero. Thus according to Chetaev's theorem [8], system (10) is unstable.

The ball capsule system is shown to be unstable from the above analysis. In fact, the unstable characteristic is crucial for the ball capsule system performance because it ensures quick response and fast closure of the ball capsule system. In addition, the ball capsule system should be robust, which means that once it is closed it should remain closed until the clutch is disengaged. If the pressure inside the chamber oscillates, the ball capsule should not open and chatter between the open and closed positions, which otherwise will adversely influence the clutch fill process. When the ball is at the open position, the minimum input pressure $P_{S}$ required to rotate the ball towards the exhaust depends on the slope angle of the capsule at the open position. Similarly, at the closed position, the minimum pressure $P_{l}$ required to hold the ball in place also depends on the capsule slope angle at the exhaust port. To avoid ball chattering, it is desirable to have different capsule slope angles. Furthermore, from the ball capsule dynamic equation (10), we can see that the dynamics of the ball capsule system can be affected by the capsule slope angle $\alpha$. In other words, the capsule slope angle $\alpha$ can be regarded as a control input to the ball capsule dynamic system. Subsequently, we propose to design the capsule shape with variable capsule slope angle $\alpha$ in order to obtain the desired performance.

\section{OPTIMAL DESIGN FOR THE BALL CAPSULE SYSTEM}

So far we have modeled and analyzed the ball capsule system dynamics. To achieve the desired ball capsule performance, the optimal design is constrained by the appropriate choice of the initial conditions $X_{\text {initial }}$ and final conditions $X_{\text {final }}$, which are chosen to meet specific design requirements. Additionally, among all the ball capsule profiles satisfying the above constraints, we would like to pick the one that has the minimum change of capsule slope angle. This will result in a smooth shape of the capsule, which in turn reduces the manufacturing cost. The total time $T_{\text {final }}$ for the ball to move from the open position to the closed position is also preferred to be as small as possible.

We define $\mathrm{N}=T_{\text {final }} / \Delta \mathrm{t}$ as the number of steps from the initial state to the final state. Note that $T_{\text {final }}$ is not predetermined, but needs to be optimized. Now we are ready to formulate the ball capsule design problem as an optimization problem that will achieve the above objectives. The cost function of the optimization problem is:

$$
\begin{aligned}
g= & \lambda_{1} \sum_{k=0}^{N}(\alpha(k)-\alpha(k-1))^{2} \Delta t+\lambda_{2}\left(X(0)-X_{\text {initial }}\right)^{2}+ \\
& \lambda_{3}\left(X(N)-X_{\text {final }}\right)^{2}+\lambda_{4}\left(T_{\text {final }}\right)
\end{aligned}
$$

In particular, the first term of the cost function ensures the increment of the capsule slope angle to be as small as possible at each time step. The last three terms ensures that the system will start from the specified initial conditions and reach the desired final conditions with minimal time $T_{\text {final }} . \lambda_{1}, \lambda_{2}, \lambda_{3}$ and $\lambda_{4}$ are the weighting factors. Consequently, the optimal control problem is to find an optimal sequence of capsule slope angle $\alpha$ to minimize the cost function while satisfying the initial and final constraints.

A systematic solution to the above optimization problem can be determined recursively via Bellman's Dynamic Programming [9-10]. Since the system model (10) is nonlinear, analytical solution cannot be obtained. Instead numerical solution will be provided. However, first we need to discretize the system model to carry out the numerical Dynamic Programming method.

The discrete state model can be written as:

$$
\begin{aligned}
v_{k}= & v_{k-1}+\frac{\Delta t}{J}\left[\left(P_{1}(k-1)-P_{2}(k-1)\right) \pi\left(r_{b} \cos \left(\alpha_{k}\right)\right)^{3}\right. \\
& \left.-F_{C}(k-1) r_{b} \sin \left(\alpha_{k}\right)\right] \\
& \theta_{k}=\theta_{k-1}+v_{k} \Delta t
\end{aligned}
$$

where $\Delta t$ refers to the sampling time interval.

In addition, since in this problem the initial condition is specified instead of the final conditions ( $T_{\text {final }}$ needs to be determined), the forward dynamic programming [11], which is a dual approach to backward dynamic programming [12] is used. The standard numerical Dynamic Programming approach requires state space discretization and interpolation, which brings in high computational burden. Thus we choose 
to implement the discrete forward dynamic programming method introduced in [11] and at the same time discretize the state space into regions, which has been presented in [13]. Consequently, with the cost function (17) and discrete model (18) and (19), the optimal design problem can be solved by using forward dynamic programming method with the capsule slope $\alpha$ as the control variable.

\section{Case Studies And Simulation Results}

In this section, we consider three possible capsule designs and provide the simulation results for comparison. The system dynamic model is constructed in Matlab/SIMULINK environment, and the parameters of the model are shown in Table 1.

TABle 1. PARAmeter VAlues of System DyNAMiC MOdel

\begin{tabular}{|c|c|c|c|}
\hline$\beta$ & $17000(\mathrm{bar})$ & $\rho_{\text {oil }}$ & $813.79\left(\mathrm{~kg} / \mathrm{m}^{3}\right)$ \\
\hline$C_{d}$ & 0.7 & $r_{\text {st }}$ & $37.3126(\mathrm{~mm})$ \\
\hline$\rho_{\text {steel }}$ & $7778.05\left(\mathrm{~kg} / \mathrm{m}^{3}\right)$ & $A_{\text {orifice }}$ & $3.0434\left(\mathrm{~mm}^{2}\right)$ \\
\hline$r_{b}$ & $1.9812(\mathrm{~mm})$ & $\omega$ & $2000(\mathrm{rpm})$ \\
\hline$P_{\text {atm }}$ & $1(\mathrm{bar})$ & $r_{c_{\_} \text {initial }}$ & $56.947(\mathrm{~mm})$ \\
\hline$A_{e x}$ & $5.8\left(\mathrm{~mm}^{2}\right)$ & $r_{c \text { final }}$ & $56.832(\mathrm{~mm})$ \\
\hline$r_{\text {cap }}$ & $2.1(\mathrm{~mm})$ & & \\
\hline
\end{tabular}

Three capsule design profiles are shown in Figure 6. The design in Figure 6(a), which is the current design of the ball capsule, has a flat surface at the ceiling and an angled wall near the exhaust to hold the ball. Figure 6(b) presents a single slope capsule design, which is analyzed in section 3. Figure 6(c) shows the optimal capsule design using Dynamic Programming method.

The input pressure $P_{S}$ used in the simulation is $1.2 \times 10^{5} \mathrm{~Pa}$. Figure 7 shows the angular velocity of the ball as it rotates towards the closed position.

\section{A. Case Study I: The Current Capsule Design}

For the current capsule design, as shown in Figure 7, the ball angular velocity jumps to a high value, and closes the exhaust quickly. But at the closed position, the ball will rotate backwards, and keep chattering between the closed position and the open position, which is undesirable for the clutch fill process. In fact, the reason that causes the chattering phenomenon is due to an improper design of the slope angles at the opening position and the closed position. The slope angle at the opening position determines the minimum input pressure $P_{s}$ (triggering pressure) required to start the ball motion toward the exhaust, and the slope angle at the closed position determines the minimum input pressure $P_{s}$ (holding pressure) required to hold the ball at the exhaust. Figure 8 shows the triggering pressure and holding pressure as a function of the slope angle. We can see that a relatively small input pressure can trigger the start of the ball motion toward the exhaust if the capsule slope angle at the opening position is small ( 0 degree for current design). However, if the slope angle at the closed position is large (36.73 degrees for current design), then the small input pressure will not be enough to hold the ball at the exhaust, which causes chattering and is exactly the problem associated with the current capsule design.

\section{B. Case Study II: The Capsule Design with Single Slope Angle}

For the capsule with single slope angle, the ball angular velocity goes up quickly as shown in Figure 7. The angular velocity reaches its peak value before closing off the exhaust. Once the capsule is closed, the ball will be held at the closing position. Therefore this design avoids the chattering problem.

Although the single slope capsule avoids the chattering problem when the input pressure is constant, it does not ensure robust performance. As shown in Figure 8, the difference between the triggering pressure and the holding pressure is insignificant. Therefore, if there are some perturbations in the input pressure Ps, the ball will oscillate between the open and closed positions. Consequently, to add control means to the ball angular velocity and also improve system robustness, the capsule design with multiple slope angles is proposed.

\section{Case Study III: The Capsule Design with Multiple Slope Angles}

To ensure robust performance, the capsule angles at the opening position and at the exhaust need to be designed appropriately. The capsule angle at the closed position (Region $\mathrm{C}$ in Figure (6c)) is designed to be smaller than that at the open position (Region A in Figure (6c)). As shown in Figure (8), this design will raise the gap between minimum triggering pressure and the minimum holding pressure, and thus increase system robustness. In addition, to avoid noise and wears at the exhaust, the impact speed at the exhaust is designed to be $200 \mathrm{rad} / \mathrm{sec}$, which is another constraint in our optimal design. Furthermore, to make manufacturing easier, the number of different slope angles should be as small as possible. Based on the above constraints, we apply the Dynamic Programming method to obtain the optimal values for the slope angles and the length of each angled section as shown in Figure 6(c).

For the capsule design shown in Figure 6(c), the ball angular velocity increases initially in region $\mathrm{A}$. When the ball enters region $\mathrm{B}$, the angular velocity decreases. Finally, the ball enters region $\mathrm{C}$, and then stops at the exhaust. The final angular velocity can be controlled by designing the slope angles. As shown in Figure 7, the ball is held at the exhaust, 
which is ensured by proper design of the slope angles at the open and closed positions.
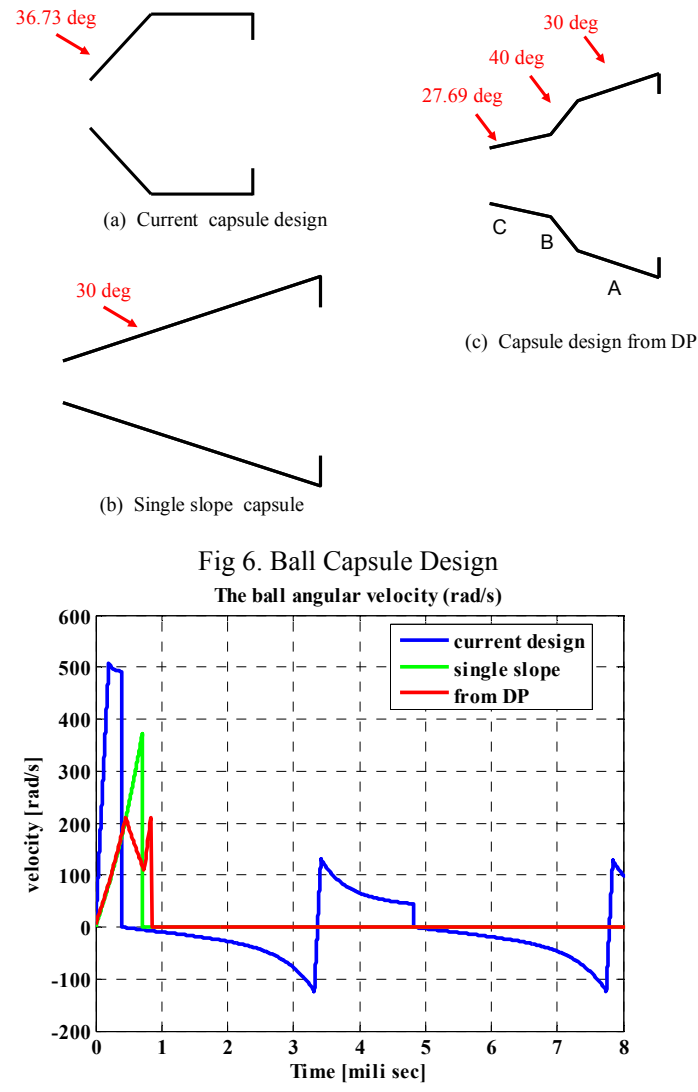

Fig 7. Angular velocity of the ball

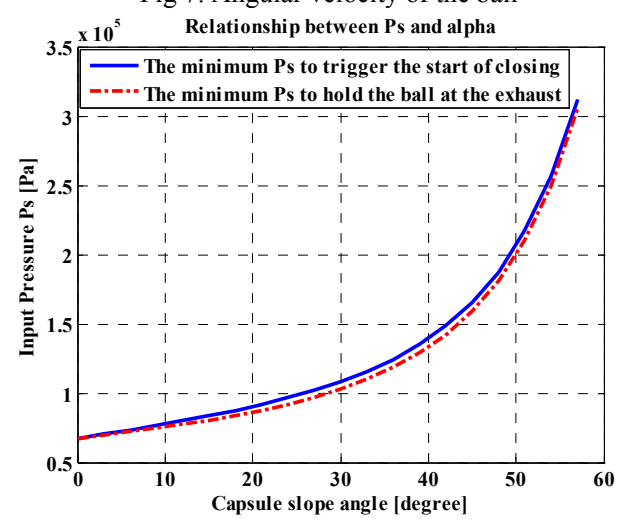

Fig 8. The closing and holding input pressure

\section{CONCLUSION}

This paper presents the modeling, analysis, and optimal design of the ball capsule system for automotive transmissions. A dynamic model of the ball capsule is first constructed. Stability analysis of the system is then conducted and reveals that the ball capsule is an unstable system, which is a critical feature for its intended purpose. To further optimize the design of the ball capsule, we formulated the problem as a constrained optimization problem and solved it using Dynamic Programming method. Simulation results show the effectiveness of the proposed design methods.

\section{ACKNOWLEDGMENTS}

The authors would like to thank GM Research and Development Center for the financial support.

\section{REFERENCES}

1 Wagner, G., "Application of Transmission Systems for Different Driveline Configurations in Passenger Cars". SAE Technical Paper 2001-01-0882.

2 Sun, Z. and Kumar, H., "Challenges and Opportunities in Automotive Transmission Control". Proceedings of 2005 American Control Conference, Portland, OR, USA, June 8-10, 2005.

3 Hebbale, K.V. and Kao, C.-K., "Adaptive Control of Shifts in Automatic Transmissions". Proceedings of the 1995 ASME International Mechanical Engineering Congress and Exposition, San Francisco, CA, 1995.

4 Bai, S., Moses, R.L, Schanz, Todd and Gorman, M.J. "Development of A New Clutch-to-Clutch Shift Control Technology". SAE Technical Paper 2002-01-1252.

5 Marano, J.E, Moorman, S.P., Whitton, M.D., and Williams, R.L. "Clutch to Clutch Transmission Control Strategy". SAE Technical Paper 2007-01-1313.

6 Han, W. and Yi, S.J., "A Study of Shift Control Using the Clutch Pressure Pattern in Automatic Transmission." Proc. Instn Mech. Engrs vol. 217 Part D: J. Automobile Engineering. Volume 217, Number 4/2003, pp 289-298.

7 Manring, N. (2005), Hydraulic Control Systems, John Wiley \& Sons, INC. Hoboken, New Jersey.

8 Khalil, H. K. (2002). Nonlinear Systems (Third Edition). Pearson Education, Inc, New Jersey.

9 Bellman, R. E. (1957), Dynamic Programming. Princeton University Press, New Jersey.

10 Bellman, R. E. and Dreyfus, S. E. (1962), Applied Dynamic Programming. Princeton University Press, New Jersey.

11 John, H.S., Lapidus, L., "Aspects of the Forward Dynamic Programming Algorithm." I\&EC Process Design and Development, 1968.

12 Larson, R. E. and Casti, J. L. (1978). Principles of Dynamic Programming, MARCEL DEKKER Inc. New York and Basel.

13 Song, X., Zulkefli, A., Sun, Z. and Miao, H., "Transmission Clutch Fill Control Using A Customized Dynamic Programming Method". Proceedings of the 2008 ASME Dynamic System and Control Conference, Ann Arbor, Michigan, 2008. 